\title{
Melnikov's Criteria and Chaos Analysis in the Nonlinear Schrödinger Equation with Kerr Law Nonlinearity
}

\author{
Jiuli Yin, Liuwei Zhao, and Lixin Tian \\ Nonlinear Scientific Research Center, Faculty of Science, Jiangsu University, Zhenjiang, \\ Jiangsu 212013, China \\ Correspondence should be addressed to Jiuli Yin; yjl@ujs.edu.cn
}

Received 27 January 2014; Accepted 30 April 2014; Published 10 June 2014

Academic Editor: Guanglu Zhou

Copyright (C) 2014 Jiuli Yin et al. This is an open access article distributed under the Creative Commons Attribution License, which permits unrestricted use, distribution, and reproduction in any medium, provided the original work is properly cited.

The dynamics of the nonlinear Schrödinger equation with Kerr law nonlinearity with two perturbation terms are investigated. By using Melnikov method, the threshold values of chaotic motion under periodic perturbation are given. Moreover we also study the effects of the parameters of system on dynamical behaviors by using numerical simulation. The numerical simulations, including bifurcation diagram of fixed points, chaos threshold diagram of system in three-dimensional space, maximum Lyapunov exponent, and phase portraits, are also plotted to illustrate theoretical analysis and to expose the complex dynamical behaviors. In particular, we observe that the system can leave chaotic region to periodic motion by adjusting controller $e$, amplitude $d_{1}$, and frequency $\omega_{2}$ of external forcing which can be considered a control strategy, and when the frequenciesy $\omega_{2}$ and $\omega_{1}$ approach the maximum frequency of disturbance, the system turmoil intensifies and control intensity increases.

\section{Introduction}

Fiber-optic signal transmission has a very wide application in the real life, which makes our lives more convenient and quicker. This is known to all. The fiber-optic signal plays an important role in real life. Optical solitary waves have been the subject of intense current research, which is motivated by their important applications in the areas of high-capacity fiber telecommunications and all-optical switches due to their capability of propagating over a long distance without attenuation and changing their shapes [1].

As is well known, the fiber-optic signal plays an important role in real life. It is the optical soliton propagation to maintain shape, amplitude and velocity constant light pulses for a long time. The use of optical solitons can achieve ultralong-distance, large-capacity optical communications.

Fiber-optic signals make it seem that the signal propagation cannot exist in pure environment. It is always influenced by external environmental perturbations. It seems that chaos may be unavoidable with external perturbations and has been observed in many practical applications such as engineering, biology, industry, and production. Besides, many other systems with external perturbations have been widely investigated by using analytic methods and numerical simulations [2-5].

However, there is little to be subject to external disturbances as a model of optical signals. In fact, the fiber-optic signal propagation will be affected by lots of interferences in the process of real propagation. Moreover, the dynamic characteristics of nonlinear system in two-frequency interference will be richer and more complicated. We therefore think it is worthwhile to undertake a detailed discussion for system (1) in order to point out which range of parameter corresponds to certain behavior. Consequently, the twofrequency disturbance has more practical meaning.

One of the famous fiber-optic models is the external periodic perturbations of the nonlinear Schrödinger equation with Kerr law nonlinearity [6]

$$
\begin{aligned}
i u_{t} & +u_{x x}+\alpha|u|^{2} u+i\left[\gamma_{1} u_{x x x}+\gamma_{2}|u|^{2} u_{x}+\gamma_{3}\left(|u|^{2}\right)_{x} u\right] \\
& =f(x, t)
\end{aligned}
$$

where $\gamma_{1}$ is the third-order dispersion and $\gamma_{2}$ and $\gamma_{3}$ are the nonlinear dispersion. Here $\gamma_{i}(i=1,2,3)$ are real parameters 
and $\alpha$ is a positive constant, $f(x, t)=e^{-i \Omega t}\left(d_{1} \cos \omega_{1}(x-c t)+\right.$ $\left.d_{2} \cos \omega_{2}(x-c t)\right)$, where the new variables $d_{i}, w_{i}(i=1,2)$ denote the amplitude and the frequency of the parametric excitation, respectively. Here $d_{i}$ and $w_{i}(i=1,2)$ are real parameters. For this reason, $d_{i}, w_{i} \geq 0(i=1,2)$.

Equation (1) describes the propagation of optical solitons in nonlinear optical fibers that exhibits a Kerr law nonlinearity. Equation (1) has an important application in various fields such as semiconductor materials, optical fiber communications, plasma physics, fluid and solid mechanics [6-12], and many other applications, can readily be described by the model, or analogous ones. Equation (1) has received more attention [6-10,13, 14]. For example, by the first integral method, Taghizadeh et al. obtained the new exact solutions of the perturbed NLSE with Kerr law nonlinearity [6]. Wiggins [12] used the $\left(G^{\prime} / G\right)$-expansion method to construct traveling wave solutions for NLSEs. Masemola et al. [9] obtained new solutions of nonlinear partial differential equation by a direct algebraic method. Miao and Zhang [11] constructed exact solutions of perturbed nonlinear Schrödinger's equation with Kerr law nonlinearity by the simplest equation method.

The study of perturbed nonlinear Schrödinger's equation with Kerr law nonlinearity is of fundamental and even practical interest. Eminent characteristics of perturbed nonlinear Schrödinger's equation with Kerr law nonlinearity have a rich content of nonlinear properties which are suitable for a detailed investigating of various dynamical states.

In this paper, we will study the chaotic behavior and control of (1) under two-frequency perturbations. We study the bifurcation and dynamical behaviors depending on the parameters by using bifurcation and chaos theories in [1521] and numerical simulation. By applying Melnikov method $[15,20,21]$, we prove the criterion of existence of chaos under periodic perturbation. Our interests have the following two points.

(i) What if there appears a system chaos under the twofrequency interference? If chaos appears, how does one design a controller to suppress chaos owing to the complex nonlinear item of system? Compared with the Duffing system $[4,5,12]$, it is easy to see that there is no damping in our system. Once perturbed with external forcing, the system may easily tend to the chaotic state. Therefore, we will select the controller which has the same function with the damping and we consider the following equation:

$$
\begin{aligned}
i u_{t}+ & u_{x x}+\alpha|u|^{2} u+i\left[\gamma_{1} u_{x x x}+\gamma_{2}|u|^{2} u_{x}+\gamma_{3}\left(|u|^{2}\right)_{x} u\right] \\
& =f(x, t)-e u_{x},
\end{aligned}
$$

where $e$ denotes the controller's strength.

(ii) We also study the effects of the parameters of system on dynamical behaviors by using numerical simulation. The numerical simulations, including bifurcation diagram of fixed points, chaos threshold diagram of system in three-dimensional space, maximum Lyapunov exponent, and phase portraits, are also plotted to illustrate theoretical analysis and to expose the complex dynamical behaviors. In particular, we observe that the system can leave chaotic region to periodic motion by adjusting controller $e$, amplitude $d_{1}$, and frequency $\omega_{2}$ of external forcing which can be considered a control strategy.

This paper is organized as follows. In Section 2, we briefly describe the fixed points and phase portraits for the two types of unperturbed system of (1). In Section 3, the conditions of existence of chaos under periodic perturbation resulting from the homoclinic bifurcations are performed. The numerical investigations are given in Section 4. The bifurcation analysis is given in Section 5. Finally, we give remark to conclude this paper in Section 6.

\section{Theoretic Analysis of System}

2.1. The NLSE with Kerr Law Nonlinearity Equation. Assume that (1) has traveling wave solutions in the form [6]

$$
u(x, t)=\phi(\xi) \exp (-i \Omega t), \quad \xi=x-c t,
$$

where $c$ is the propagation speed of a wave $(c>0)$.

Substituting (3) into (1), we get

$$
\begin{gathered}
i\left(\gamma_{1} \phi^{\prime \prime \prime}+\gamma_{2} \phi^{2} \phi^{\prime}+2 \gamma_{2} \phi^{2} \phi^{\prime}-c \phi^{\prime}\right)+\left(\Omega \phi+\phi^{\prime \prime}+\alpha \phi^{3}\right) \\
=d_{1} \cos \left(w_{1} \xi\right)+d_{2} \cos \left(w_{2} \xi\right),
\end{gathered}
$$

where $\gamma_{i}(i=1,2,3), \alpha$, and $k$ are positive constants and prime meaning differentiation with respect to $\xi$.

By virtue of [6], we have

$$
\gamma_{1} \phi^{\prime \prime}-c \phi+A \phi^{3}=d_{1} \cos \left(w_{1} \xi\right)+d_{2} \cos \left(w_{2} \xi\right),
$$

where $A=(1 / 3) \gamma_{2}+(2 / 3) \gamma_{3}$ denote the parametric of linear and nonlinear terms. Equation (5) is the fiber-optic signal transmission system in ideal environment. But it seems that the signal propagation cannot exist in pure environment. It is always influenced by external environmental perturbations.

Without loss of generality in (5), we set $\gamma_{1}=1$. By the transformations $\phi=x_{1}, \phi^{\prime}=x_{2}$, (6) can be transformed into first-order nonautonomous equation:

$$
\begin{aligned}
& x_{1}^{\prime}=x_{2}, \\
& x_{2}^{\prime}=c x_{1}-A x_{1}^{3}+d_{1} \cos \left(w_{1} \xi\right)+d_{2} \cos \left(w_{2} \xi\right) .
\end{aligned}
$$

2.2. Fixed Points and Phase Portraits for Unperturbed System. If $d_{1}=d_{2}=0$, (6) is considered an unperturbed system and can be written as

$$
\begin{aligned}
& x_{1}^{\prime}=x_{2}, \\
& x_{2}^{\prime}=c x_{1}-A x_{1}^{3} .
\end{aligned}
$$

The system (7) is a Hamiltonian system with Hamiltonian function

$$
H\left(x_{1}, x_{2}\right)=\frac{1}{2} x_{2}^{2}-\frac{1}{2} c x_{1}^{2}+\frac{1}{4} A x_{1}^{4} .
$$




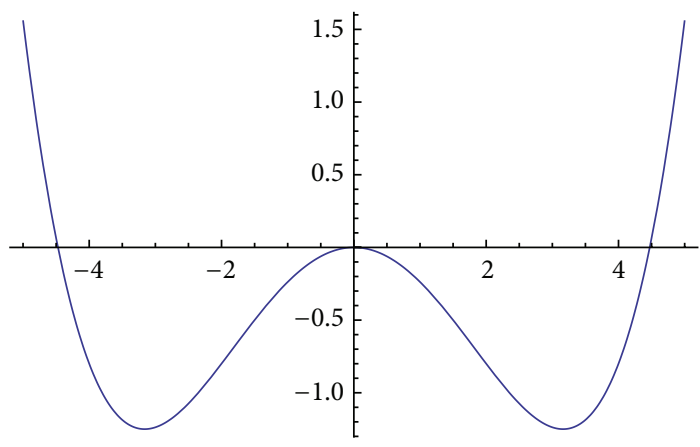

(a)

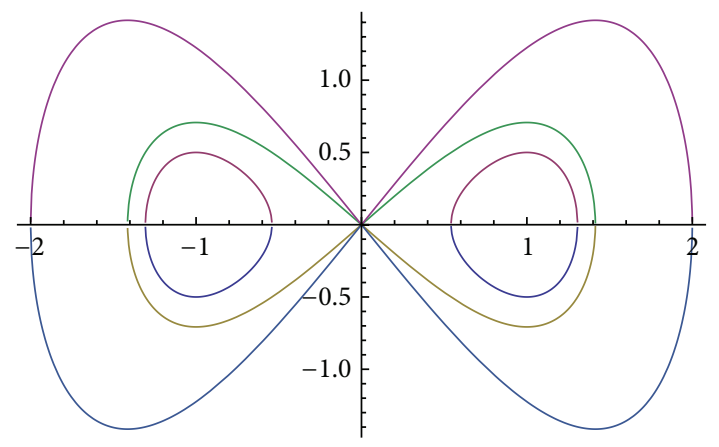

(b)

FIgURE 1: (a) The two-well potential function of the system; (b) the corresponding phase space portraits.

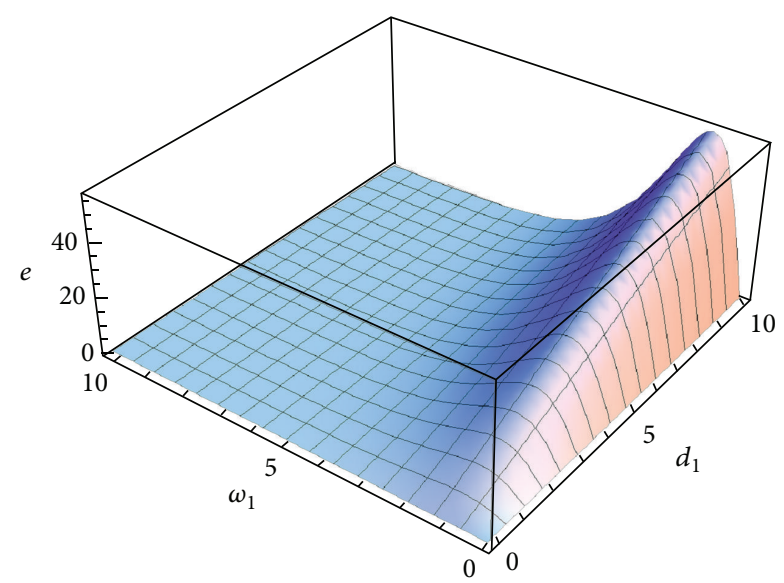

FIgURE 2: Chaotic threshold in $\left(d_{1}, w_{1}, e\right)$ space.

And the function

$$
V\left(x_{1}\right)=-\frac{1}{2} c x_{1}^{2}+\frac{1}{4} A x_{1}^{4}
$$

is called the potential function. By the analysis of the fixed points $\left(x_{1_{j}}, x_{2_{j}}\right)$ and their stabilities for system (7), we can easily obtain the following results.

Lemma 1. When $c A>0$, the unperturbed system has three equilibrium points: two centers $(\sqrt{c / A}, 0)$ and $(-\sqrt{c / A}, 0)$ and one saddle $(0,0)$. The saddle is connected to itself by two symmetric homoclinic orbits as shown in Figure 1(b).

\section{Melnikov Theoretic Analysis}

In this section, we discuss the chaotic behaviors of (6). Melnikov theory has proved to be a simple, elegant, and successful alternative to characterizing the complex dynamics of multistable oscillators. This section, thus, develops a global analysis technique, known as Melnikov's method, to find the necessary conditions for homoclinic bifurcation to occur. For a detailed derivation of Melnikov's method, there are several

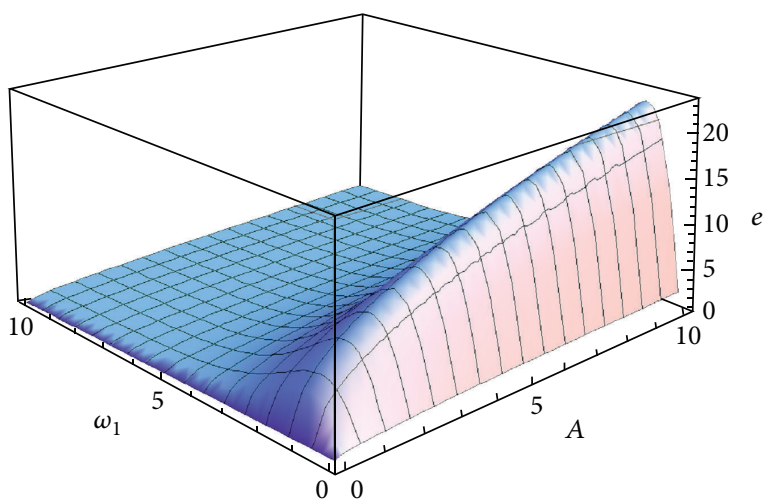

Figure 3: Chaotic threshold in $\left(\omega_{1}, A, e\right)$ space.

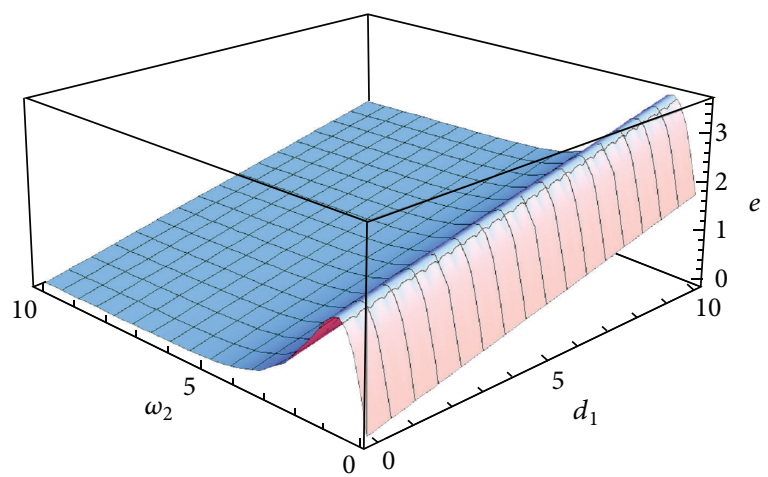

FIGURE 4: Chaotic threshold in $\left(\omega_{2}, d_{1}, e\right)$ space.

texts of varying rigor and sophistication to which the reader is referred [12, 22-24].

In this section, we discuss the chaotic behaviors of (6) in which $d_{1}$ and $d_{2}$ are assumed to be small parameters. Transformations of $d_{1} \rightarrow \varepsilon d_{1}$ and $d_{2} \rightarrow \varepsilon d_{2}$ are done in order to apply the $\varepsilon$ first-order perturbation scheme of the Melnikov theory. Hence, the system of (6) may be written as

$$
\begin{aligned}
& x_{1}^{\prime}=x_{2}, \\
& x_{2}^{\prime}=c x_{1}-A x_{1}^{3}+\varepsilon d_{1} \cos \left(\phi_{1}\right)+\varepsilon d_{2} \cos \left(\phi_{2}\right),
\end{aligned}
$$




$$
\begin{aligned}
& \phi_{1}^{\prime}=\omega_{1}, \\
& \phi_{2}^{\prime}=\omega_{2} .
\end{aligned}
$$

3.1. Melnikov Criterion for Chaos. The unperturbed system for system (7) has homoclinic orbits. When the perturbation is added, the closed homoclinic orbits break and may have transverse homoclinic or heteroclinic orbits. By the SmaleBirkhoff Theorem [12, 25], the existence of such orbits results in chaotic dynamics. We therefore apply the Melnikov method to system (7) for finding the criteria of the existence of homoclinic or heteroclinic bifurcation and chaos.

The Melnikov method derives a function to describe the first order distance between perturbed stable and unperturbed manifolds. Suppose that the unperturbed homoclinic or heteroclinic orbit is written as $\left(x_{1}, x_{2}\right)=\left(x_{1}(\xi), x_{2}(\xi)\right)$. Satisfying the conditions for a double-well potential gives rise to a homoclinic orbit in the phase space for $\varepsilon=0$. The homoclinic trajectory can be found by setting $H(a, b)=$ 0 . Solving for the resulting displacement and differentiating to determine velocity, the homoclinic trajectory is given as follows:

$$
\begin{aligned}
& \left(x_{1}, x_{2}\right) \\
& =\left(\sqrt{\frac{2 c}{A}} \operatorname{sech}(\sqrt{c} \xi),-\sqrt{\frac{2 c}{A}} \sqrt{c} \operatorname{sech}(\sqrt{c} \xi) \tanh (\sqrt{c} \xi)\right),
\end{aligned}
$$

and then the Melnikov function for system (7) can be given by

$$
\begin{aligned}
& M\left(\xi_{0}\right) \\
& \quad=\int_{-\infty}^{\infty} x_{2}(\xi)\left[d_{1} \cos w_{1}\left(\xi+\xi_{0}\right)+d_{2} \cos w_{2}\left(\xi+\xi_{0}\right)\right] d \xi
\end{aligned}
$$

where $\xi_{0}$ is the cross-section time of the Poincare map and $\xi_{0}$ can be interpreted as the initial time of the forcing term.

Because it is difficult to give analytical expression of $x_{2}(\xi)$, we will compute $x_{2}(\xi)$ numerically in Section 5 . We note that $x_{2}(\xi)$ is a function of time from $+\infty$ to $-\infty$. We therefore choose that the initial condition is $\xi=0$ and $x_{2}(\xi)$ would be an odd function of time for the homoclinic orbit and an even function for heteroclinic orbit.

For the homoclinic orbits $\Gamma_{\mathrm{ho}}^{ \pm}$, the Melnikov function can be simplified as

$$
\begin{aligned}
& M_{1}\left(\xi_{0}\right) \\
& \quad=\int_{-\infty}^{\infty} x_{2}(\xi)\left[d_{1} \cos w_{1}\left(\xi+\xi_{0}\right)+d_{2} \cos w_{2}\left(\xi+\xi_{0}\right)\right] d \xi \\
& \quad=-2 d_{1} \sin w_{1} \xi_{0} \int_{0}^{\infty} x_{2}(\xi) \sin w_{1} \xi d \xi
\end{aligned}
$$

$$
\begin{aligned}
& -2 d_{2} \sin w_{2} \xi_{0} \int_{0}^{\infty} x_{2}(\xi) \sin w_{2} \xi d \xi \\
= & -2 d_{1} \sin w_{1} \xi_{0} I_{\mathrm{hom} 1}\left(w_{1}\right)-2 d_{2} \sin w_{2} \xi_{0} I_{\mathrm{hom} 2}\left(w_{2}\right),
\end{aligned}
$$

where $I_{\text {hom } 1}=\int_{0}^{\infty} x_{2}(\xi) \sin w_{1} \xi d \xi$ and $I_{\text {hom } 2}=$ $\int_{0}^{\infty} x_{2}(\xi) \sin w_{2} \xi d \xi$ are functions of the frequencies $w_{1}$ and $w_{2}$, respectively.

Using the previous results and Melnikov's theorem [26, 27], the following is stated: if $M_{1}\left(\xi_{0}\right)=0$ and $M_{1}^{\prime}\left(\xi_{0}\right) \neq 0$ for some $\xi_{0}$ and some set of parameters, then horseshoes exist and chaos occurs $[12,25]$. If $M_{1}\left(\xi_{0}\right)$ has a simple zero and the corresponding critical parameter value is

$$
(e)_{0}=\frac{\left|d_{1} I_{\mathrm{hom} 1}\left(\omega_{1}\right)+d_{2} I_{\mathrm{hom} 2}\left(\omega_{2}\right)\right|}{B},
$$

where $B=\int_{0}^{\infty} x_{2}^{2}(\xi) d \xi$ is a constant once $x_{2}(\xi)$ is given, then in the system with fractional order displacement (11) the deterministic chaos may appear for certain parameter values which satisfy the relation

$$
e<(e)_{0}
$$

Remark 2. Using the Melnikov criterion for the appearance of the intersection between the perturbed and unperturbed separatrices, therefore, for fixed frequencies $\omega_{1}$ and $\omega_{2}$, the system always produces Smale commutation of chaos.

3.2. Control of Chaos. Because the fiber-optic transmission system in the chaotic state is very sensitive to its initial condition and chaos often causes irregular behavior, chaos is undesirable. It is not hard to see that system (6) is similar to Duffing system, except the absence of damping in the former. Therefore, we will select the controller that has the same function with the damping.

Equation (2) is equivalent to the following system:

$$
-c x_{1}+x_{1}^{\prime \prime}+A x_{1}^{3}=d_{1} \cos \left(\omega_{1} \xi\right)+d_{2} \cos \left(\omega_{2} \xi\right)-e x_{1}^{\prime}
$$

Equation (16) can be transformed into first-order nonautonomous equation:

$$
\begin{aligned}
& x_{1}^{\prime}=x_{2} \\
& x_{2}^{\prime}=c x_{1}-A x_{1}^{3}+\varepsilon d_{1} \cos \left(w_{1} \xi\right)+\varepsilon d_{2} \cos \left(w_{2} \xi\right)-\varepsilon e x_{2}
\end{aligned}
$$

where $d_{1} \rightarrow \varepsilon d_{1}, d_{2} \rightarrow \varepsilon d_{2}$, and $e \rightarrow \varepsilon e$. by

Now, the Melnikov function for system (17) can be given

$$
\begin{aligned}
M_{1}\left(\xi_{0}\right)=\int_{-\infty}^{\infty} x_{2}(\xi)[ & -e x_{2}(\xi)+d_{1} \cos \omega_{1}\left(\xi+\xi_{0}\right) \\
& \left.+d_{2} \cos \omega_{2}\left(\xi+\xi_{0}\right)\right] d \xi
\end{aligned}
$$




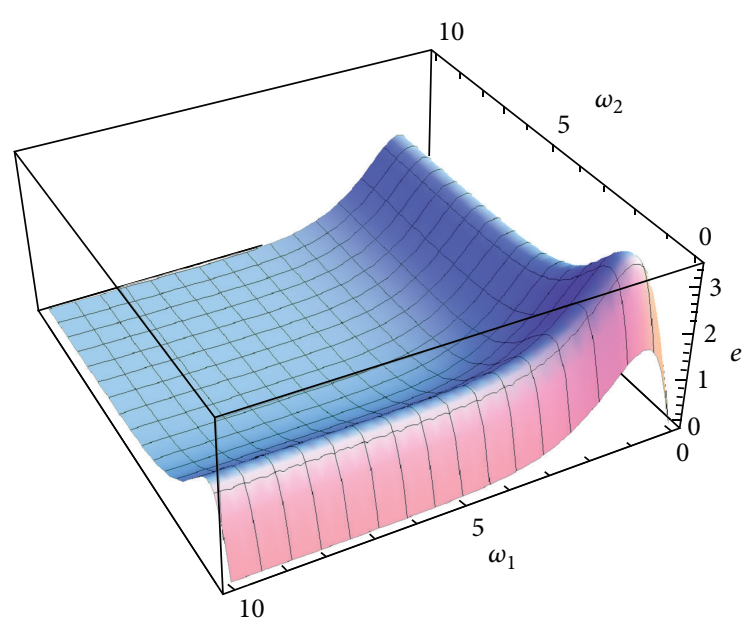

FIGURE 5: Chaotic threshold in $\left(\omega_{1}, \omega_{2}, e\right)$ space.

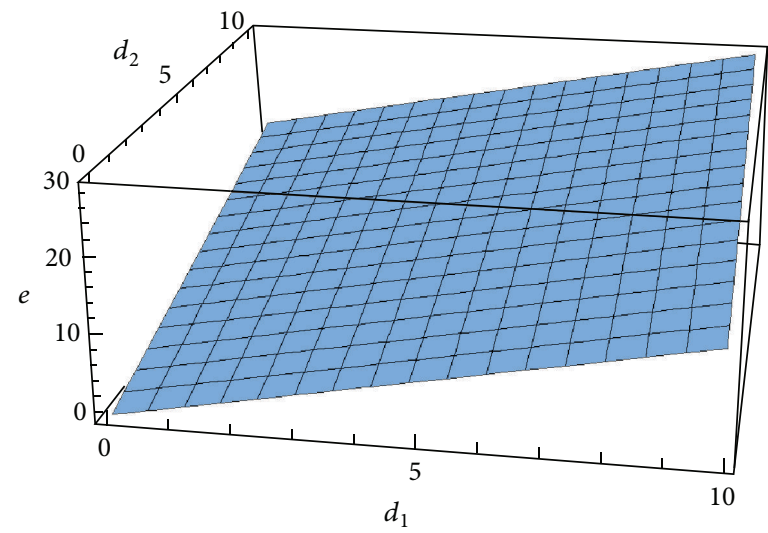

Figure 6: Chaotic threshold in $\left(d_{1}, d_{2}, e\right)$ space.

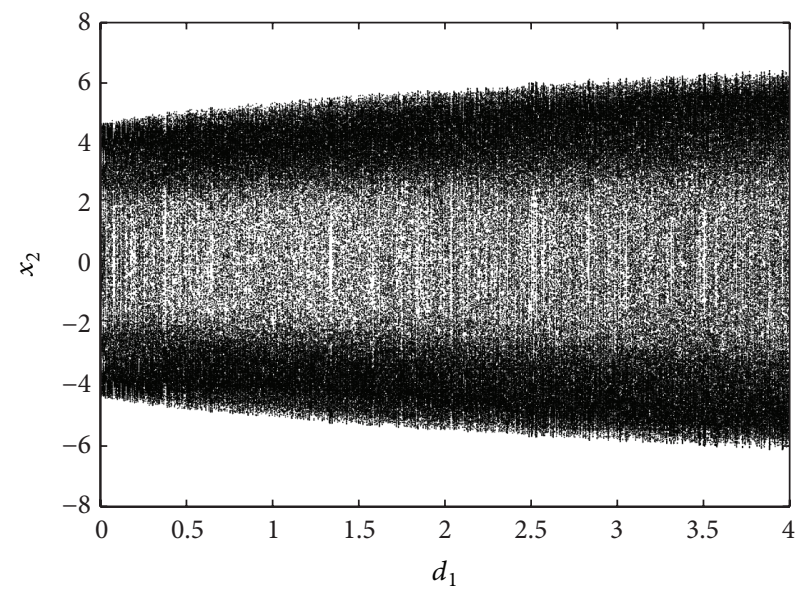

(a)

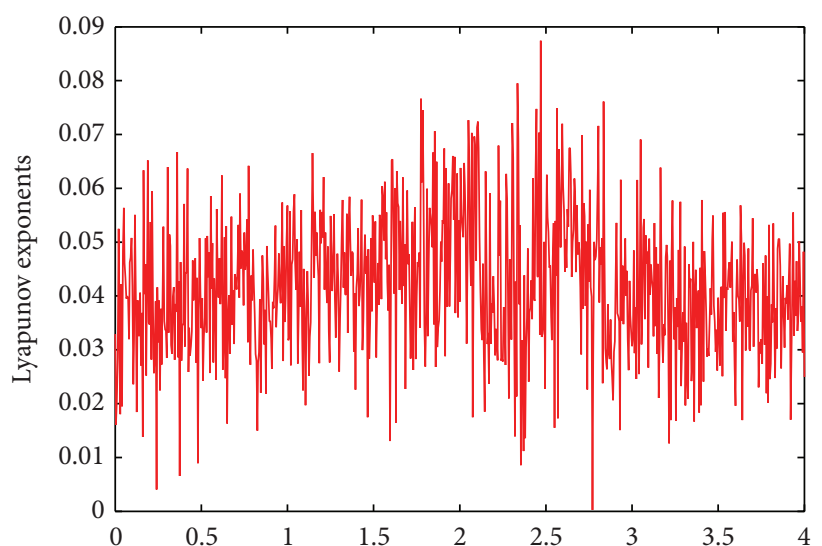

(b)

Figure 7: (a) Bifurcation diagram of system (6) in $\left(d_{1}, x_{2}\right)$ plane $0 \leq d \leq 4$. (b) Maximum Lyapunov exponents corresponding to (a). 


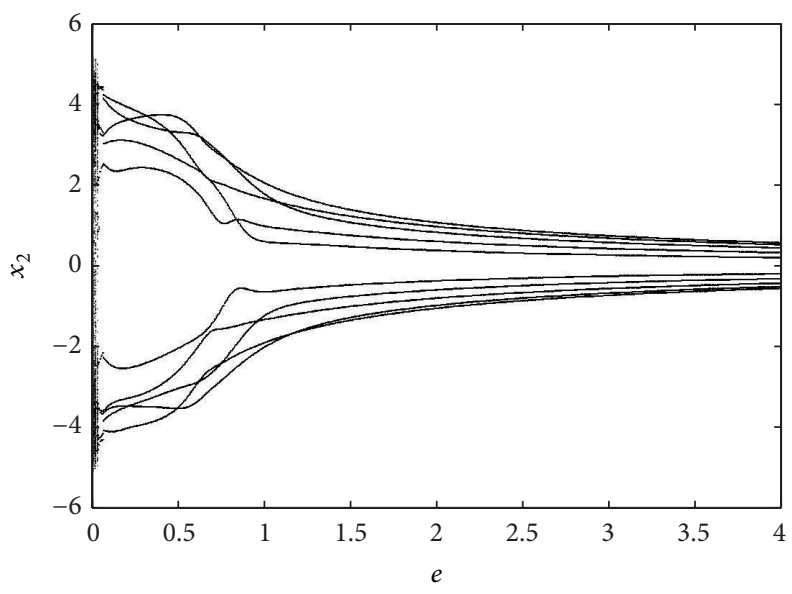

(a)

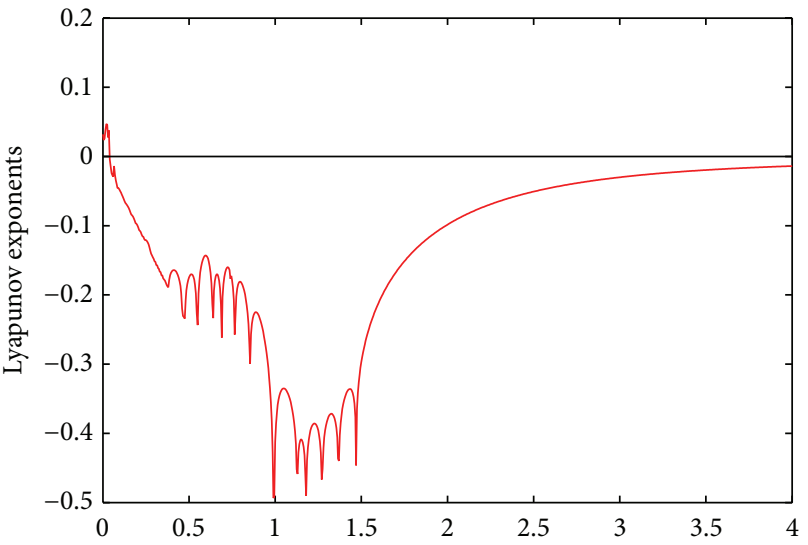

(b)

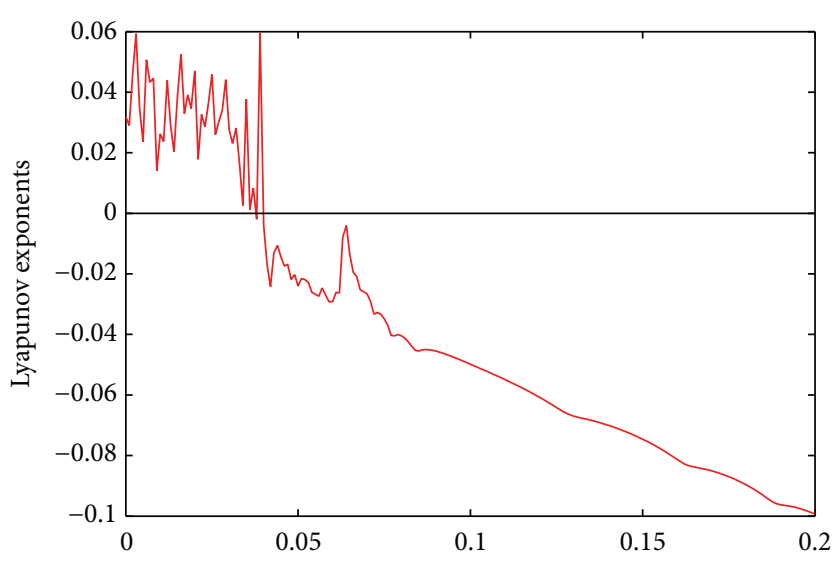

(c)

Figure 8: (a) Bifurcation diagram of system (17) in (e, $\left.x_{2}\right)$ plane $0 \leq e \leq 4$. (b) Maximum Lyapunov exponents corresponding to (a). (c) Enlarged view of (b).

and the Melnikov function can be simplified as

$$
\begin{aligned}
M_{1}\left(\xi_{0}\right) & \\
= & -2 e \int_{0}^{\infty} x_{2}^{2}(\xi) d \xi-2 d_{1} \sin w_{1} \xi_{0} \int_{0}^{\infty} x_{2}(\xi) \sin w_{1} \xi d \xi \\
& -2 d_{2} \sin w_{2} \xi_{0} \int_{0}^{\infty} x_{2}(\xi) \sin w_{2} \xi d \xi \\
= & -2 e B-2 d_{1} \sin w_{1} \xi_{0} I_{\text {hom } 1}\left(w_{1}\right) \\
& -2 d_{2} \sin w_{2} \xi_{0} I_{\text {hom } 2}\left(w_{2}\right) .
\end{aligned}
$$

Thus, if

$$
e<\frac{\left|d_{1} I_{\text {hom } 1}\left(\omega_{1}\right)+d_{2} I_{\text {hom } 2}\left(\omega_{2}\right)\right|}{B} \equiv R(\omega),
$$

then there is a $\overline{\xi_{0}}$ such that $M_{1}\left(\overline{\xi_{0}}\right)$ and $\left.\left(\partial M_{1} / \partial \xi_{0}\right)\right|_{\xi=\xi_{0}} \neq 0$, $\overline{\xi_{0}} \in\left[0,2 \pi / \omega_{1}\right]$, and the following lemma can be obtained.
Lemma 3. The homoclinic bifurcation will occur at

$$
e=R(\omega)
$$

Remark 4. For (20) the distance between the stable and unstable manifolds of the homoclinic point $(0,0)$ is zero and the manifolds intersect transversely forming the transverse homoclinic orbits. The presence of such orbits implies that, for certain parameters (21) and the countable infinity of unstable periodic orbits, an uncountable set of bounded nonperiodic orbits and a dense orbit are the main characteristics of the chaotic motion.

\section{Numerical Simulations}

In this section, we give numerical simulations to support the theoretical results obtained in the previous sections and to find other new dynamics.

The interesting problem is to analyze the parameter regions for optical fiber signals' stable propagation of the controlled system. The controlled fiber-optic transmission system has several parameters and each of them plays different 


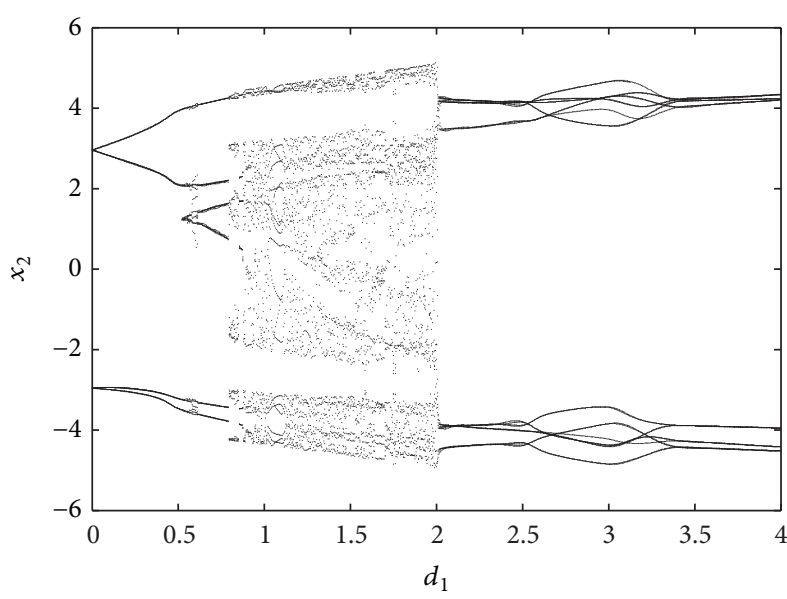

(a)

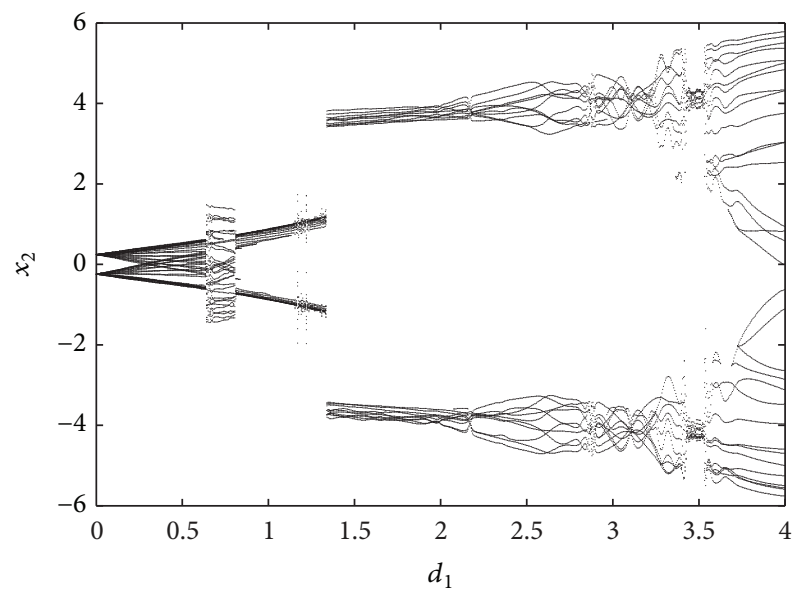

(c)

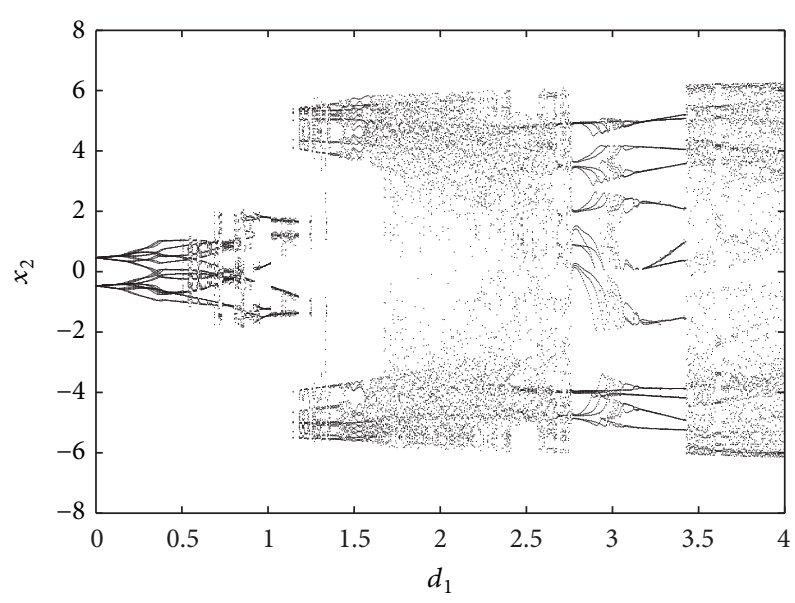

(b)

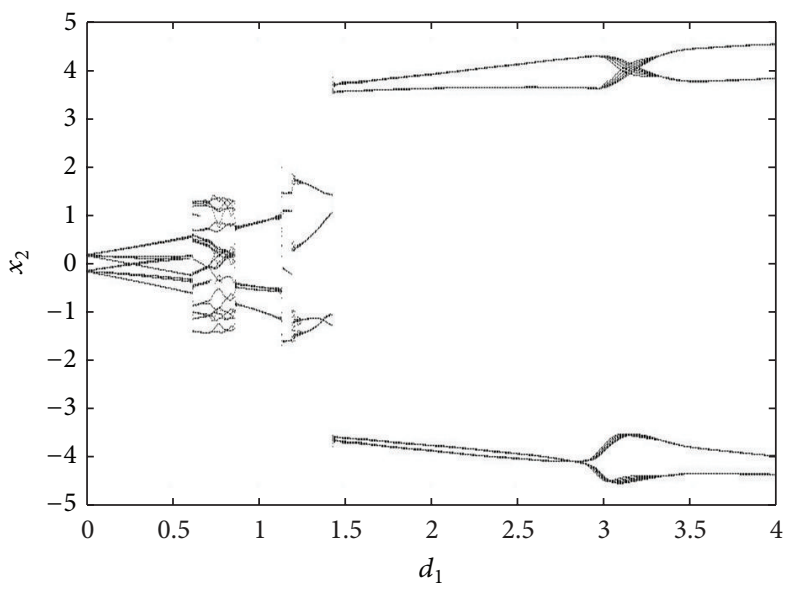

(d)

FIgURE 9: Bifurcation diagrams of system (17) in $\left(d_{1}, x_{2}\right)$ plane for case (iii): (a) $\omega_{2}=0.8$, (b) $\omega_{2}=1.8$, (c) $\omega_{2}=2.5$, and (d) $\omega_{2}=3$.

and virtual roles in the system. We will analyze the influence on optic-fiber signals propagation of controlled system (21) when the parameter of system changes with the fixed controller.

Let us study the intersections of the invariant manifolds of the saddle point. It is known that these intersections are the necessary conditions for the existence of chaos. Since the Melnikov function theory measures the distance between the perturbed stable manifold and unstable manifolds, a homoclinic tangency will occur when a real solution can be found for some time $\xi_{0}$ such that the function has a simple zero. This means that only necessary conditions for the appearance of strange attractors are obtained from PoincaréMelnikov-Arnold analysis, and therefore one always has the chance of finding sufficient conditions for the elimination of even transient chaos. Then the general necessary condition for which the invariant manifolds intersect is given by

$$
e=R(\omega) \text {. }
$$

We give numerical simulations to support the theoretical results as shown in Figures 2-6.
The chaotic threshold in $\left(d_{1}, \omega_{1}, e\right)$ space with $d_{2}=1$, $\omega_{2}=0.08, A=1$, and $c=0.5$ is shown in Figure 2. The chaotic threshold in $\left(A, \omega_{1}, e\right)$ space with $d_{2}=1, \omega_{2}=0.08$, $d_{1}=1.2$, and $c=0.5$ is shown in Figure 3 . The chaotic threshold in $\left(\omega_{2}, d_{1}, e\right)$ space with $d_{2}=1, \omega_{1}=0.05, A=1.2$, and $c=1$ is shown in Figure 4 . The chaotic threshold in $\left(\omega_{2}, \omega_{1}, e\right)$ space with $d_{2}=1, d_{1}=1.2, A=0.5$, and $c=0.8$ is shown in Figure 5. The chaotic threshold in $\left(d_{2}, d_{1}, e\right)$ space with $A=$ $0.5, \omega_{2}=1, \omega_{1}=1.2$, and $c=0.8$ is shown in Figure 6 . When the value of $e$ is below the surface, the system may be chaotic state. In order to show what happens to the solutions and attractors as one crosses these bifurcation surfaces, we chose the parameter values from the regions $e<(e)_{0}$, and then the system (17) can exhibit chaos.

Remark 5. This implies that if $\varepsilon>0$ is sufficiently small, the reduced equation (17) has transverse homoclinic orbits resulting in possible chaotic dynamic. With $c$ and $A$ constant, we study chaotic threshold as a function of only the frequency parameter $\omega$. A typical plot of $e$ is shown in Figure 2. The qualitative form of this function remains the same as $c$ and 


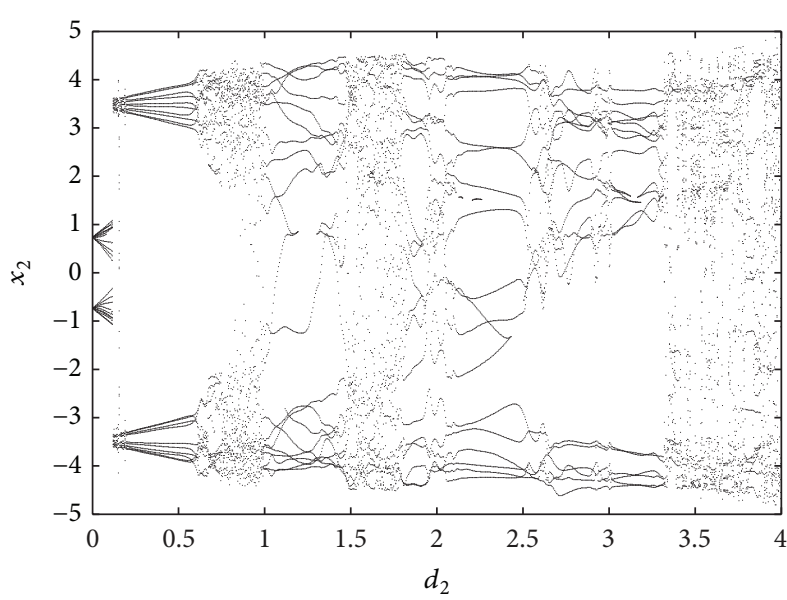

(a)

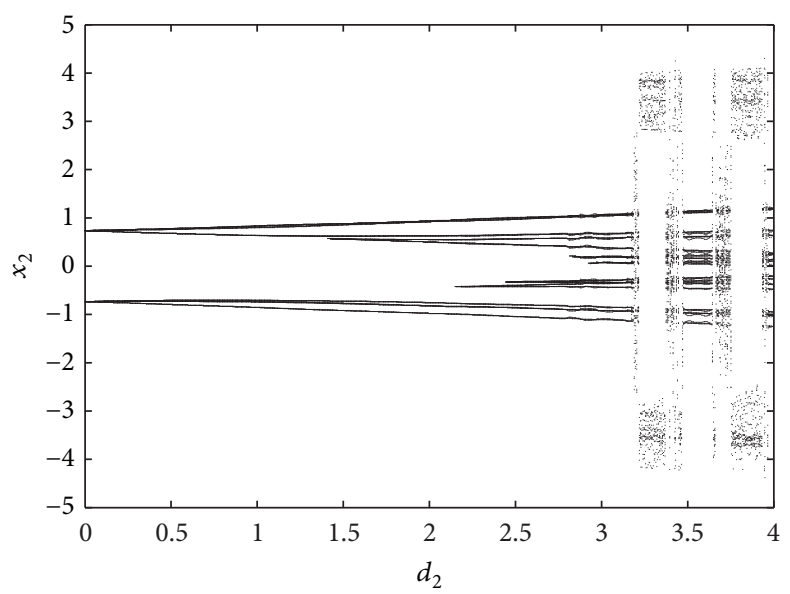

(c)

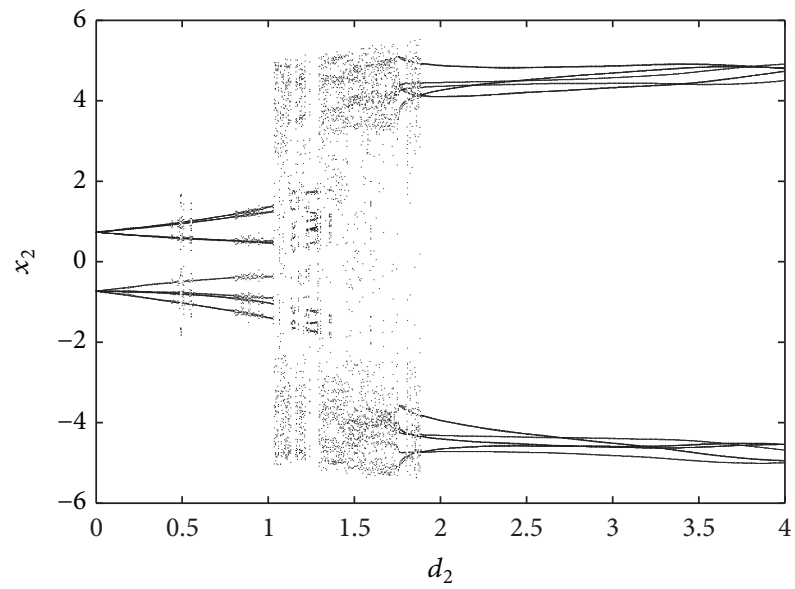

(b)

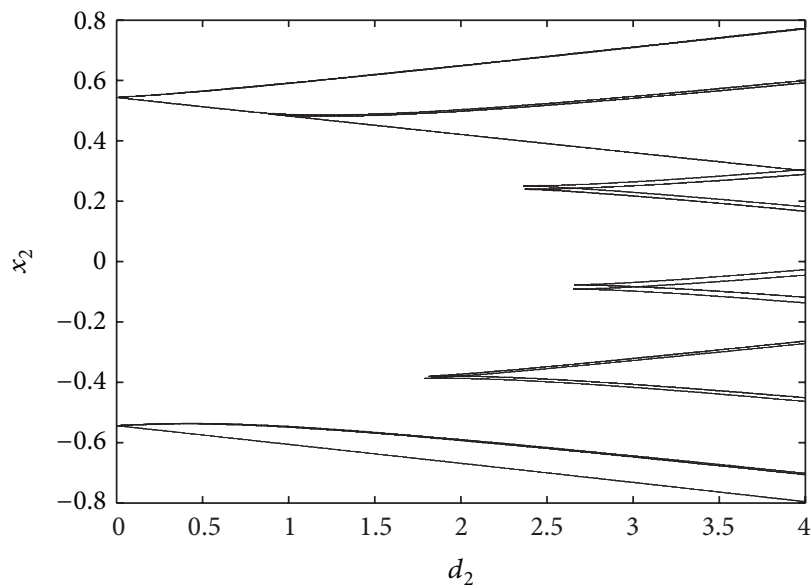

(d)

FIgURE 10: Bifurcation diagrams of system (17) in $\left(d_{2}, x_{2}\right)$ plane for case (iii): (a) $\omega_{2}=0.7$, (b) $\omega_{2}=1.5$, (c) $\omega_{2}=3.2$, and (d) $\omega_{2}=4$.

$A$ are the values for which the potential is two-well. Another point is that, when the ratio $e$ tends to zero, this means that the external amplitude $d_{1}$ tends to infinity and then the Melnikov theory is not valid for these values.

\section{Bifurcation Analysis}

In this section, we give numerical simulations to support the theoretical results of control of chaos.

5.1. Perturbation and Controlled System Analysis. We will discuss the behaviors of the fiber-optic signal transmission under perturbation and we draw the bifurcation diagram of $(6)$ in $\left(d_{1}, x_{2}\right)$ plane and the corresponding maximal Lyapunov exponents in Figure 7. System (6) is integrated using the Runge-Kutta technique of order four to conduct numerical simulation. Numerical calculations have been made for the selected parameter values $A=0.2, c=0.0001$, $d_{2}=1.5, \omega_{1}=1.2$, and $\omega_{2}=1$ with the initial conditions $x_{1}=1$ and $x_{2}=0$. Since the equation is nonlinear, its solution therefore admits the possibility of periodic and chaotic orbits.

In Figure 7, we can observe that the value of Lyapunov exponents is positive (a positive top Lyapunov exponent for a bounded attractor is usually a sign of chaos), so the system easily converts to chaos even if there is small perturbation. It indicates that the system is outside perturbed, as the optical fiber transmission signal is very vulnerable to the phenomenon of chaos. So an appropriate controller is needed to satisfy the practical applications of fiber-optic propagation. Such maps can be used to suppress chaotic dynamics of the system.

Next the corresponding numerical simulations are performed for the case of periodic perturbation of system (16); we select $d=1$. In Figure 8(a) after a large band of chaotic regime for $e \in(0,0.045)$ one can find a sequence of backward period-doubling bifurcations as a route to periodic motion after a periodic regime; another bifurcation takes place at a critical value $e=0.062$ where another large band of chaotic regime with small periodic window occurs. At $e \in(0.046$, 0.061 ) and $e>0.063$, the system displays periodic behavior after backward period-doubling bifurcations. Such maps can be used to suppress chaotic dynamics of the system.

From Figure 8(b) one can see that for smaller values of controller $e$, the top Lyapunov exponent is positive. As $e$ increases, the top Lyapunov exponent changes from positive value to negative value, signifying the suppressing of 


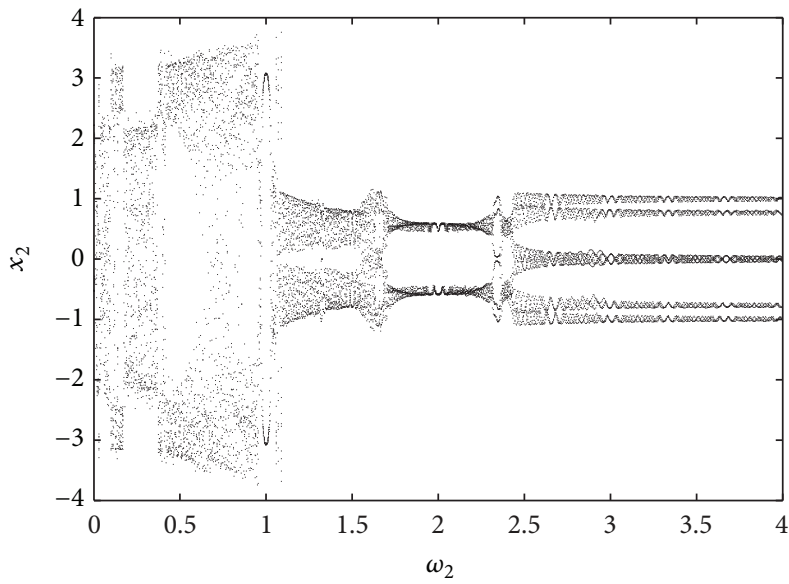

(a)

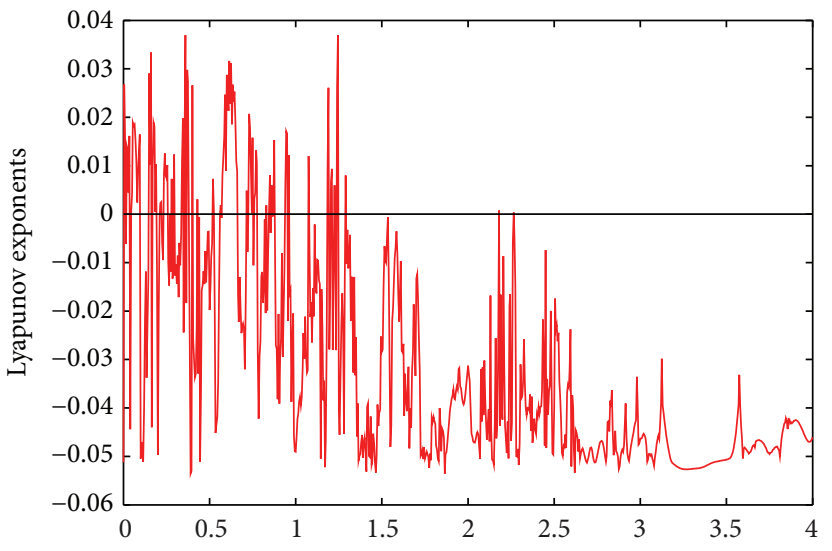

(c)

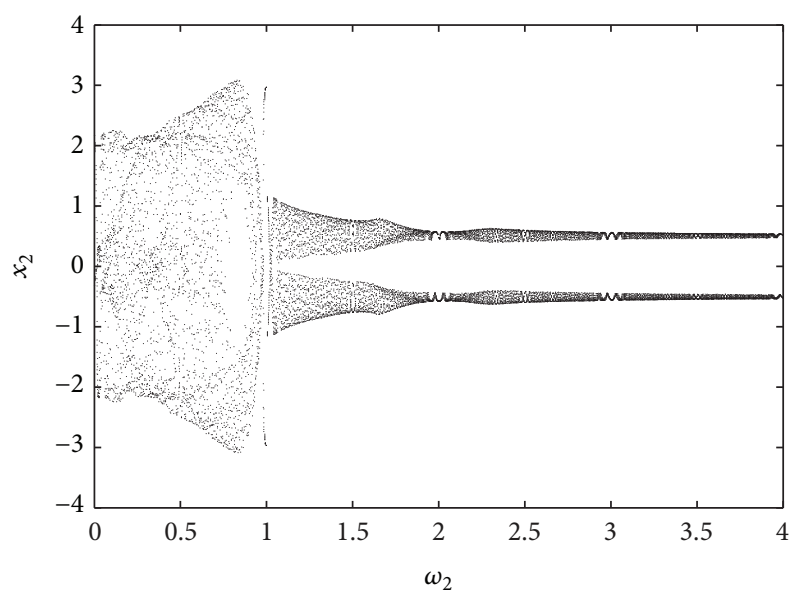

(b)

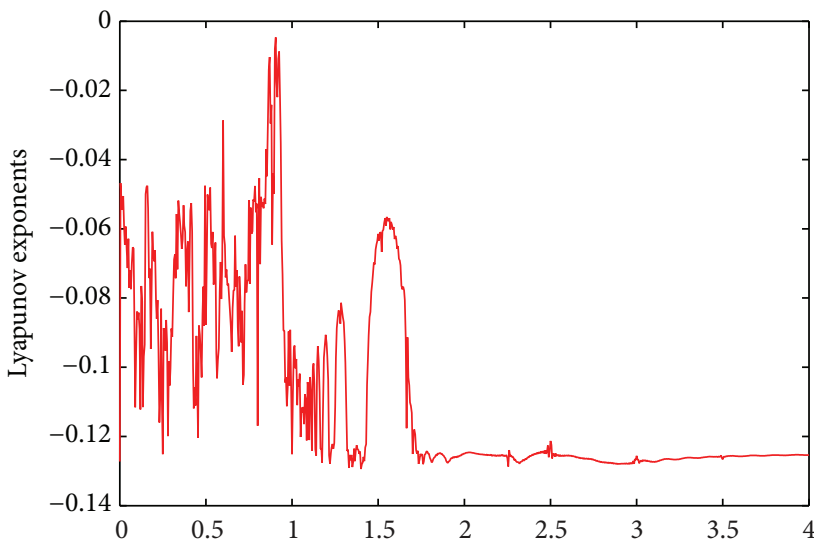

(d)

FIgURE 11: Bifurcation diagrams of system (17) in $\left(\omega_{2}, x_{2}\right)$ plane for case (iii): (a) $e=0.1$; (b) $e=0.25$. The maximum Lyapunov exponents: (c) corresponding to (a), (d) corresponding to (b).

homoclinic chaos motion. Beyond the threshold for onset of chaotic motion, there are some "periodic windows;" this can be the feature of the transient chaos. However, the larger control parameters are responsive for the decreasing signal amplitude. It is easy to see that the signal cannot propagate normally and might leak from the media, which is called escape.

Remark 6. According to the above analysis, the increasing of the controller's coefficient $e$ makes the system stable, but escape occurs when $e$ crosses a certain value.

5.2. Parameters Analysis. Now we wish to find other interesting bifurcation structures and dynamics of system (17). The bifurcation parameters are considered in the following five cases:

(i) varying $d_{1}$ in range $0<d_{1}<4$ and fixing $A=0.2$, $c=0.0001, e=0.1, \omega_{1}=1.2$, and $d_{2}=1.5$ for several values of $\omega_{2}$,

(ii) varying $d_{2}$ in range $0<d_{2}<4$ and fixing $A=0.2$, $c=0.0001, e=0.1, \omega_{1}=1.2$, and $d_{1}=1$ for several values of $\omega_{2}$, (iii) varying $\omega_{2}$ in range $0<\omega_{2}<4$ and fixing $A=0.2$, $c=0.0001, \omega_{1}=1, d_{1}=0.5$, and $d_{2}=0.5$ for several values of $e$,

(iv) varying $e$ in range $0<e<0.4$ and fixing $\omega_{2}=2$, $d_{2}=0.5, \omega_{1}=1.2, d_{1}=1.8$, and $c=0.0002$ for several values of $A$,

(v) varying $e$ in range $0<e<0.4$ and fixing $A=0.2$, $d_{1}=1, \omega_{1}=1.2, d_{2}=1.2$, and $\omega_{2}=2$ for several values of $c$.

For cases (i) and (ii), we give bifurcation diagrams in $\left(d_{1}, x_{2}\right)$ and $\left(d_{2}, x_{2}\right)$ plane of system (17) for $0<d_{1}<4$ and $0<d_{2}<4$ and several values of $\omega_{2}$ in Figures 9 and 10, respectively. We observe that a wide chaotic region in Figure 9(a). The coexistences of periodic orbits and chaotic motions in chaotic windows and intermittent mechanism in Figure 9(b). Figure 9(c) shows that the onset of chaos after period-n and chaotic regions with complex periodic windows, Figure 9(d) also shows the onset of chaos and chaotic regions with small periodic windows. Comparing Figures 10(a)-10(d), we find that, as $\omega_{2}$ is increased, the complexity of dynamical 


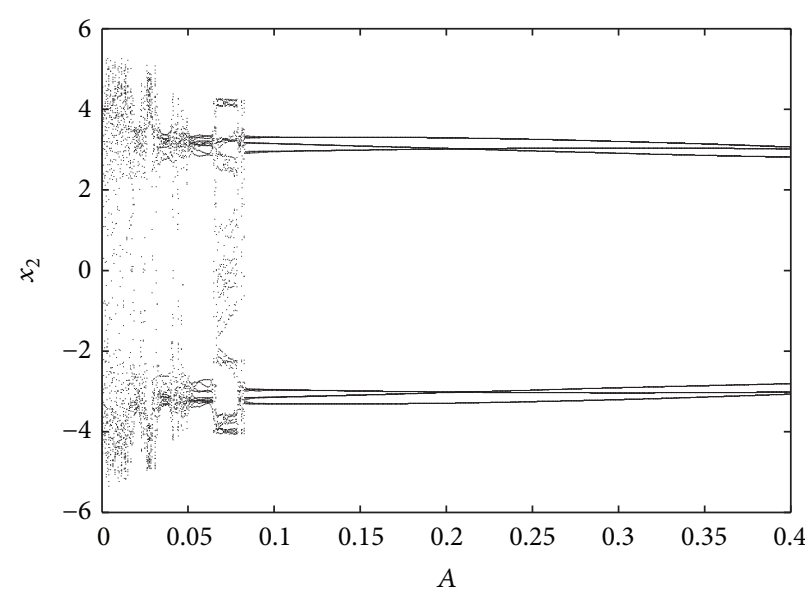

(a)

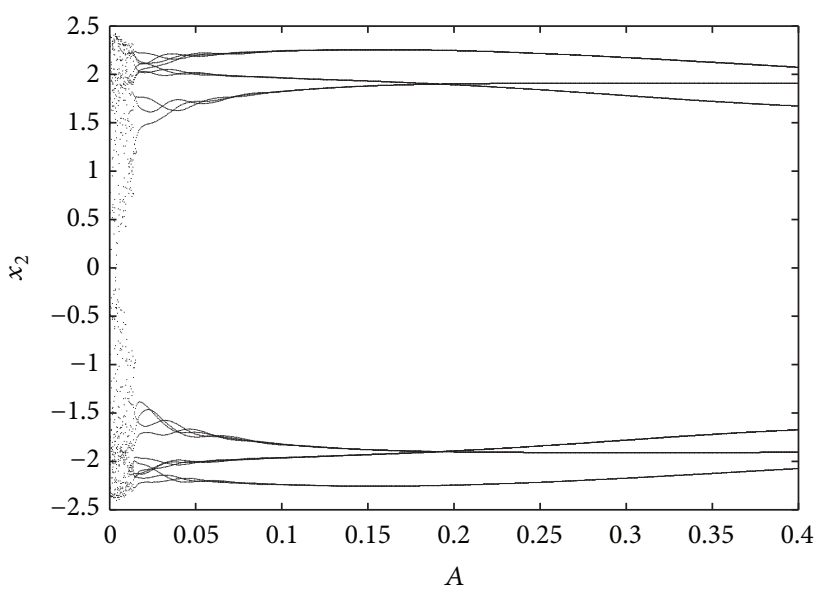

(c)

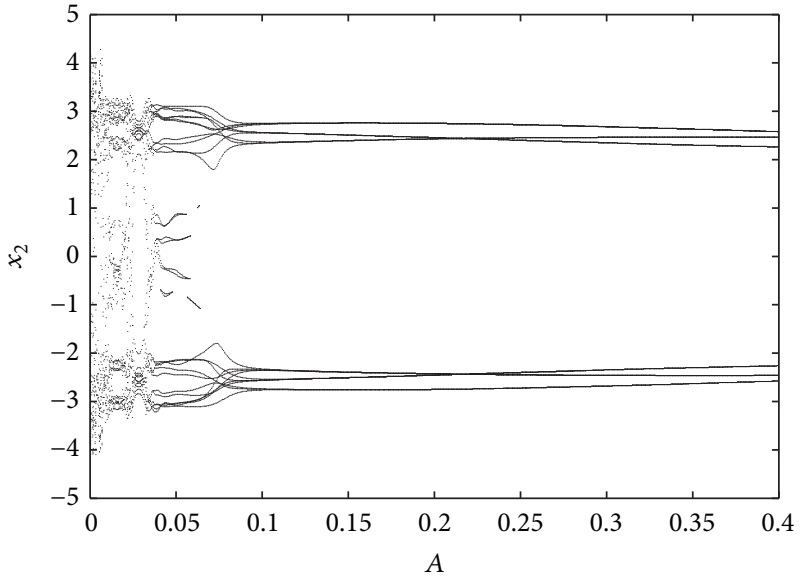

(b)

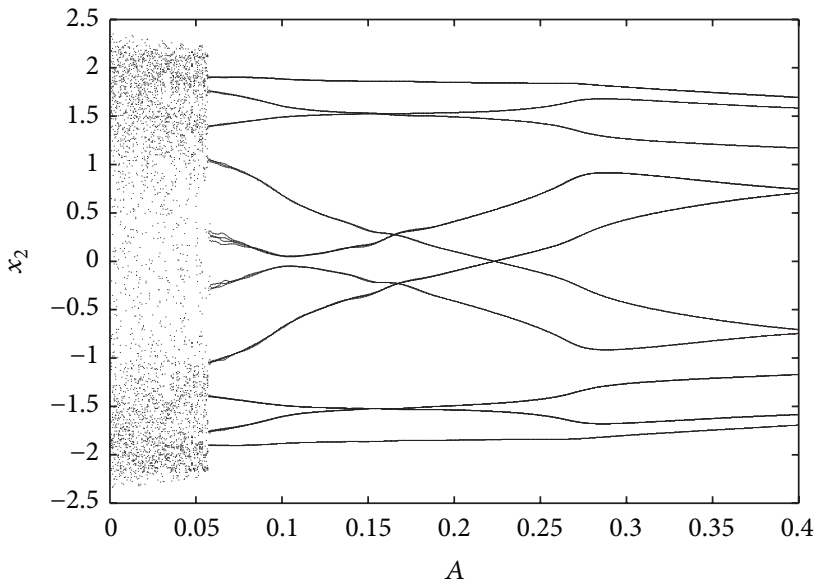

(d)

Figure 12: Bifurcation diagrams of system (17) in $\left(e, x_{2}\right)$ plane for case (iii): (a) $A=0.3$, (b) $A=0.5$, (c) $A=1$, and (d) $A=2$.

behaviors is decreased, and as $\omega_{2}=4$ there is only a periodone orbit and there is no bifurcation (see Figure 10(d)).

For case (iii), the bifurcation diagrams in $\left(\omega_{2}, x_{2}\right)$ plane and the corresponding maximum Lyapunov exponents are given in Figure 11, where we show that the maximum Lyapunov exponents are all negative in Figure 11(d) for $e=$ 0.25 which are corresponding to quasiperiodic solutions in Figure 11(b). Figures 11(a) and 11(c) exhibit the process of the quasiperiodic route to chaos and chaos converges to quasiperiodic orbit.

For case (iv), the bifurcation diagrams in Figures 12(a)$12(\mathrm{~d})$ in $\left(e, x_{2}\right)$ plane for various values of $A$ are plotted for showing the effect of parameter $A$ on dynamical behaviors. When $A$ is increased a little, the chaotic behavior occurrence has changed and the amplitude had decreased a little in Figure 12(b). But, as $A$ is further increased, there is only the periodfive orbit and a period-doubling bifurcation of periodone.

For case (v), the bifurcation diagrams in Figures 13(a)13(d) in $\left(e, x_{2}\right)$ plane for various values of $c$ are plotted for showing the effect of parameter $c$ on dynamical behaviors. We show that the chaotic behavior occurs alternately in smaller region of $c$ and the chaotic motions behavior occurs alternately in bigger region as a is increased. Therefore, we find that the parameter $c$ plays a very important role for dynamical behaviors from comparing. It can be considered as an control strategy of chaos by adjusting the parameter $e$.

\section{Conclusions}

In this paper, we have investigated the behaviors of the nonlinear Schrödinger equation with Kerr law nonlinearity with two perturbation terms and find many complex and interesting dynamical behaviors by using analytic and numerical methods. We conclude that chaos occurs easily due to the absence of damping in the system. This phenomenon will cause the distortion in the process of information transmission. One can add a controller to suppress the chaos. The efficiency of this controller was demonstrated. The complex fiber-optic transmission system of the perturbed NLSE with Kerr law nonlinearity was controlled. What is more is that we discussed the sensitivity to be controlled and found the practical parameters regions. Some of the results obtained for other NLSEs can be extended correspondingly. 


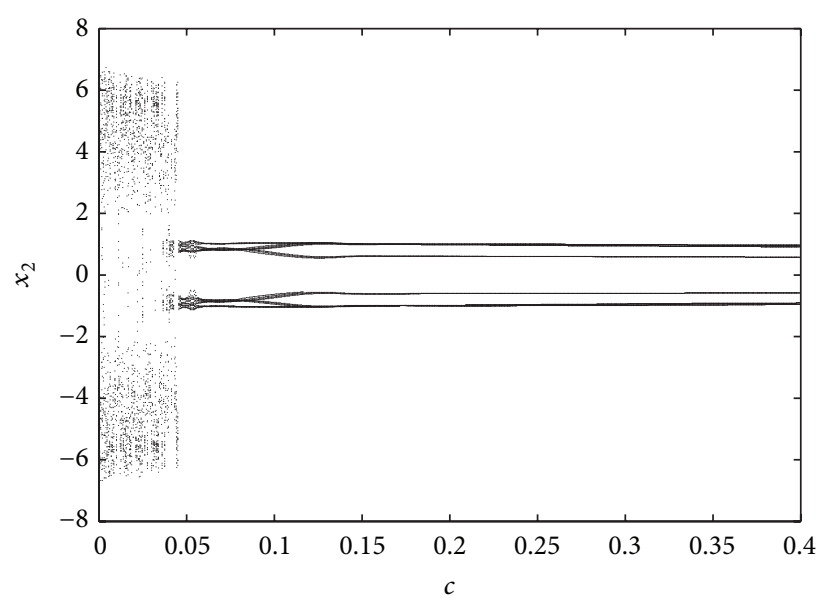

(a)

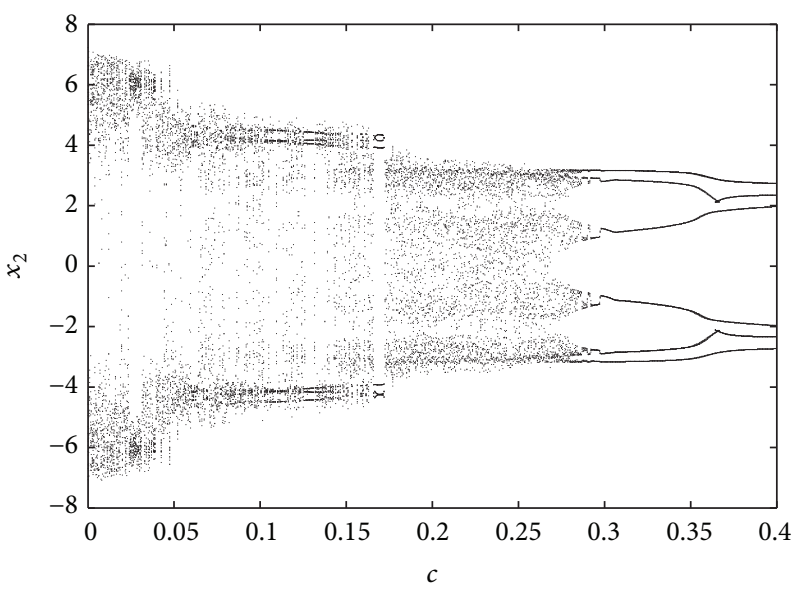

(c)

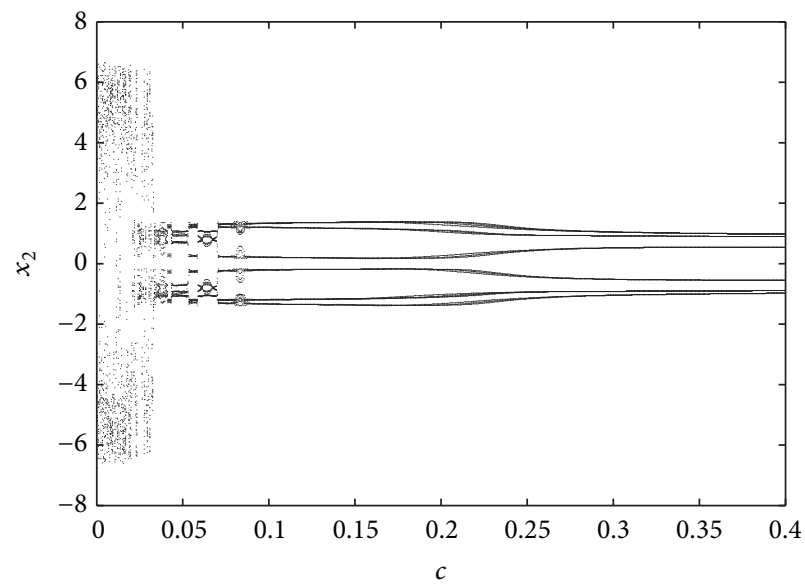

(b)

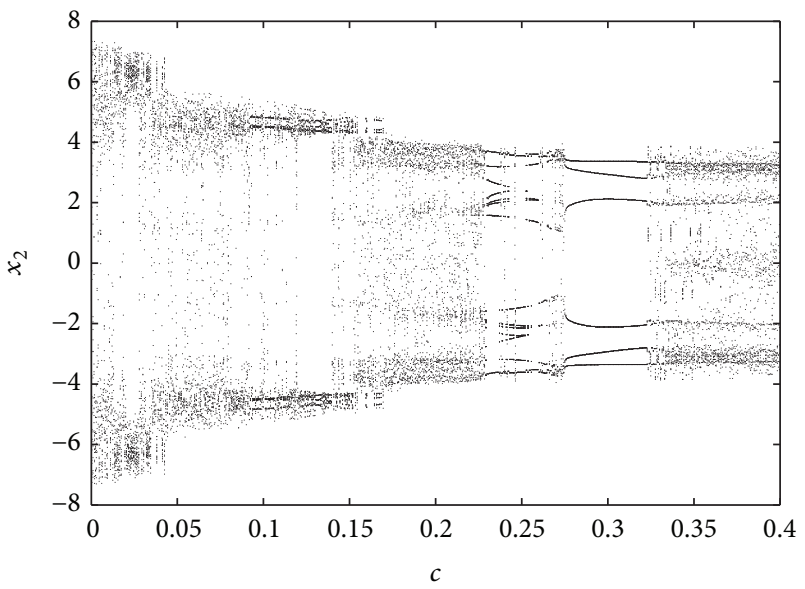

(d)

Figure 13: Bifurcation diagrams of system (17) in $\left(e, x_{2}\right)$ plane for case (iii): (a) $c=0.002$, (b) $c=0.02$, (c) $c=0.8$, and (d) $c=1.2$.

\section{Conflict of Interests}

The authors declare that there is no conflict of interests regarding the publication of this paper.

\section{Acknowledgment}

This work is supported by the National Nature Science Foundation of China (no. 11101191).

\section{References}

[1] Y. JiuLi, "A duoble optical solitary wave in a nonlinear Schrödinger-type equation," Chinese Physics B, vol. 22, no. 6, Article ID 060205, 2013.

[2] S. Özgül, M. Turan, and A. Yildirim, "Exact traveling wave solutions of perturbed nonlinear Schrödinger's equation (NLSE) with Kerr law nonlinearity," Optik, vol. 123, no. 24, pp. 22502253, 2012.

[3] Z. Jing and R. Wang, "Complex dynamics in Duffing system with two external forcings," Chaos, Solitons and Fractals, vol. 23, no. 2, pp. 399-411, 2005.
[4] Z. Jing, J. Huang, and J. Deng, "Complex dynamics in three-well duffing system with two external forcings," Chaos, Solitons and Fractals, vol. 33, no. 3, pp. 795-812, 2007.

[5] R. Wang, J. Deng, and Z. Jing, "Chaos control in duffing system," Chaos, Solitons and Fractals, vol. 27, no. 1, pp. 249-257, 2006.

[6] N. Taghizadeh, M. Mirzazadeh, and A. Mahmoodirad, "Application of Kudryashov method for high-order nonlinear Schridinger equation," Indian Journal of Physics, vol. 87, no. 8, pp. 781-785, 2013.

[7] Z.-Y. Zhang, Z.-H. Liu, X.-J. Miao, and Y.-Z. Chen, "New exact solutions to the perturbed nonlinear Schrödinger's equation with Kerr law nonlinearity," Applied Mathematics and Computation, vol. 216, no. 10, pp. 3064-3072, 2010.

[8] H. Moosaei, M. Mirzazadeh, and A. Yildirim, "Exact solutions to the perturbed nonlinear Schrödinger's equation with kerr law nonlinearity by using the first integral method," Nonlinear Analysis: Modelling and Control, vol. 16, no. 3, pp. 332-339, 2011.

[9] P. Masemola, A. H. Kara, and A. Biswas, "Optical solitons and conservation laws for driven nonlinear Schrödingers equation with linear attenuation and detuning," Optics and Laser Technology, vol. 45, no. 1, pp. 402-405, 2013.

[10] A. R. Shehata, "The traveling wave solutions of the perturbed nonlinear Schrödinger equation and the cubic-quintic 
Ginzburg Landau equation using the modified ( $\left.\mathrm{G}^{\prime} / \mathrm{G}\right)$-expansion method," Applied Mathematics and Computation, vol. 217, no. 1, pp. 1-10, 2010.

[11] X.-J. Miao and Z.-Y. Zhang, "The modified ( $\left.G^{\prime} / G\right)$-expansion method and traveling wave solutions of nonlinear the perturbed nonlinear Schrödinger's equation with Kerr law nonlinearity," Communications in Nonlinear Science and Numerical Simulation, vol. 16, no. 11, pp. 4259-4267, 2011.

[12] S. Wiggins, Introduction to Applied Nonlinear Dynamical Systems and Chaos, vol. 2 of Texts in Applied Mathematics, Springer, New York, NY, USA, 1990.

[13] W.-X. Ma and M. Chen, "Direct search for exact solutions to the nonlinear Schrödinger equation," Applied Mathematics and Computation, vol. 215, no. 8, pp. 2835-2842, 2009.

[14] J. Belmonte-Beitia and G. F. Calvo, "Exact solutions for the quintic nonlinear Schrödinger equation with time and space modulated nonlinearities and potentials," Physics Letters A: General, Atomic and Solid State Physics, vol. 373, no. 4, pp. 448453, 2009.

[15] J. Guckenheimer and P. Holmes, Nonlinear Oscillations, Dynamical Systems and Bifurcations of Vector Fields, Springer, New York, NY, USA, 1983.

[16] M. Lakshmanan and K. Murali, Chaos in Nonlinear Oscillators: Controlling and Synchronization, World Scientific, 1996.

[17] E. Ott, Chaos in Dynamical Systems, Cambridge University Press, 2002.

[18] S. N. Rasband, Chaotic Dynamics of Nonlinear Systems, John Wiley \& Sons, New York, NY, USA, 1990.

[19] J. M. T. Thompson and H. B. Stewart, Nonlinear Dynamics and Chaos, John Wiley \& Sons, New York, NY, USA, 1986.

[20] S. Wiggins, Global Bifurcation and Chaos: Analytical Methods, Springer, 1988.

[21] Y. Uyaroğlu and S. Emiroğlu, "Passivity-based chaos control and synchronization of the four dimensional Lorenz-Stenflo system via one input," Journal of Vibration and Control, 2013.

[22] J. Guckenheimer and P. Holmes, Nonlinear Oscillations, Dynamical Systems, and Bifurcations of Vector Fields, vol. 42 of Applied Mathematical Sciences, Springer, New York, NY, USA, 1983.

[23] D. W. Jordan and P. Smith, Nonlinear Ordinary Differential Equations: An Introduction for Scientists and Engineers, Oxford University Press, New York, NY, USA, 2007.

[24] A. H. Nayfeh and B. Balachandran, Introduction to Nonlinear Dynamics: Analytical, Computational, and Experimental Methods, John Wiley \& Sons, New York, NY, USA, 1995.

[25] J. Guckenheimer and P. Homel, Nonlinear Oscillations, Dynamical System, and Bifurcation of Vector Fields, Springer, 1992.

[26] S. C. Stanton, B. P. Mann, and B. A. M. Owens, "Melnikov theoretic methods for characterizing the dynamics of the bistable piezoelectric inertial generator in complex spectral environments," Physica D: Nonlinear Phenomena, vol. 241, no. 6, pp. 711720, 2012.

[27] V. K. Melnikov, "On the stability of a center for time-periodic perturbations," Trudy Moskovskogo Matematičeskogo Obščestva, vol. 12, pp. 3-52, 1963 (Russian). 


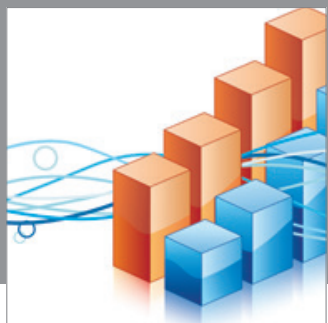

Advances in

Operations Research

mansans

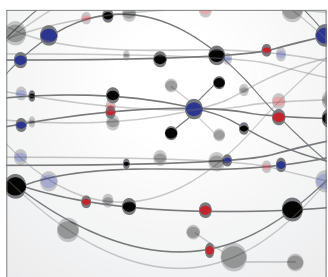

The Scientific World Journal
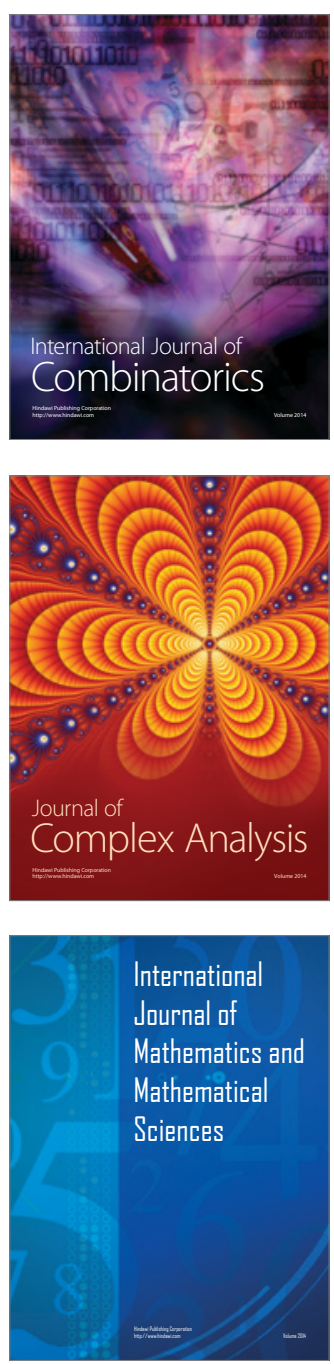
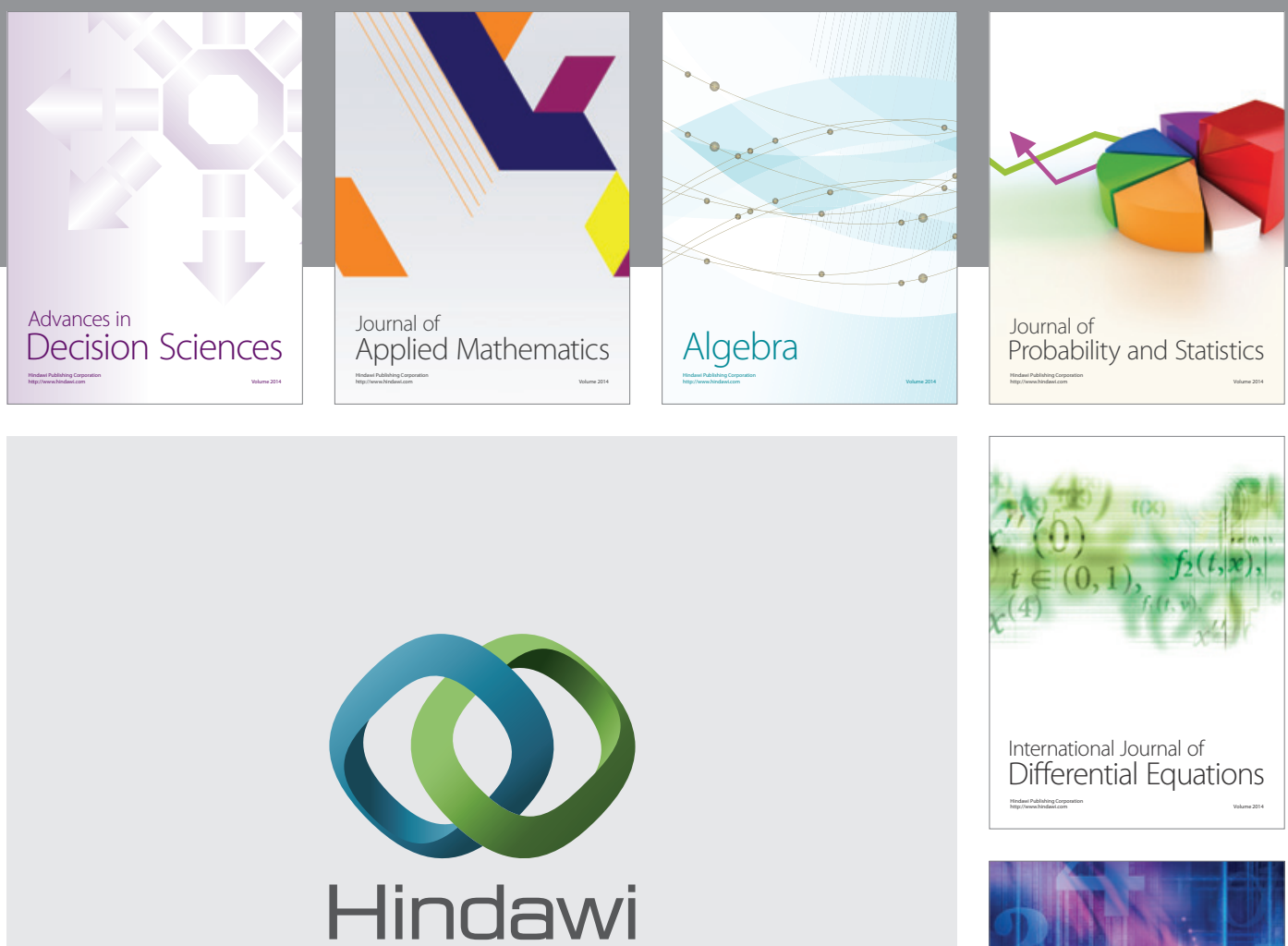

Submit your manuscripts at http://www.hindawi.com
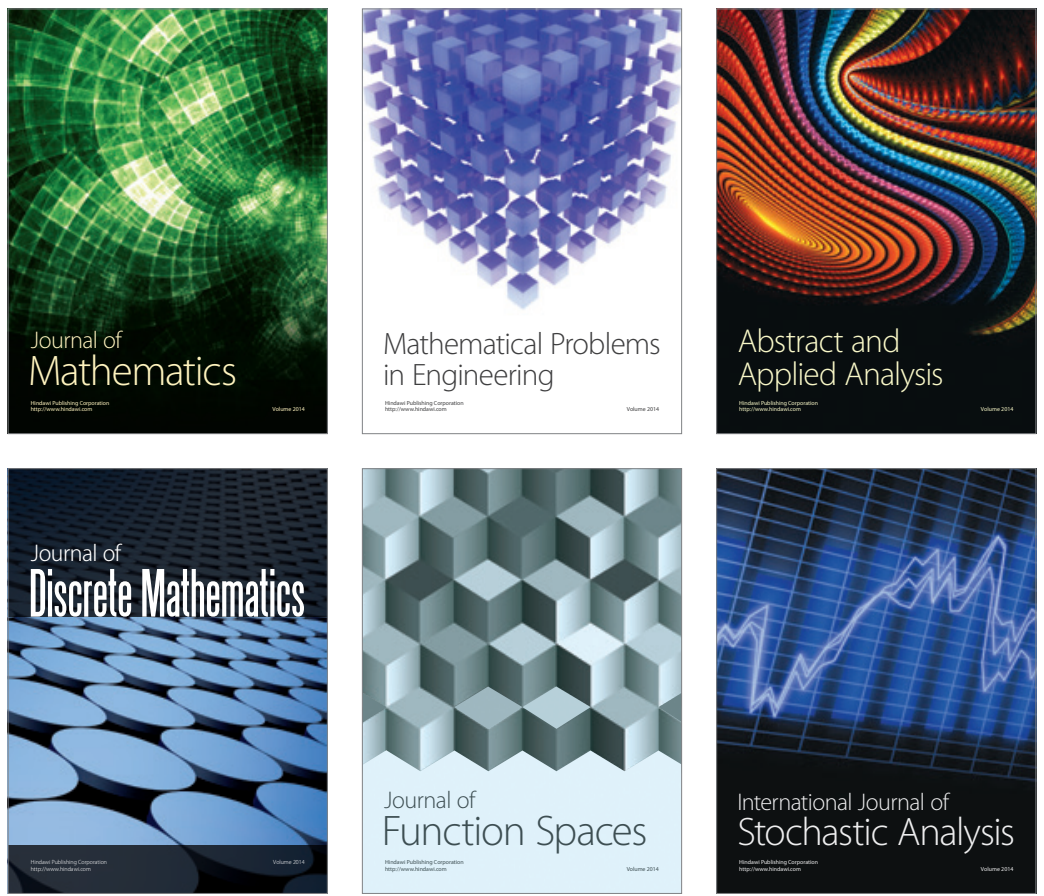

Journal of

Function Spaces

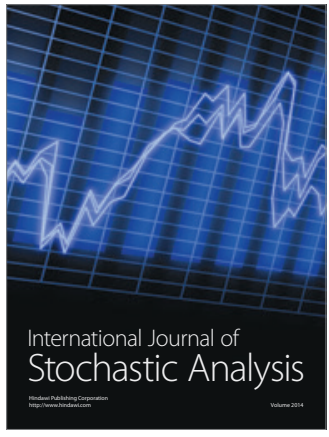

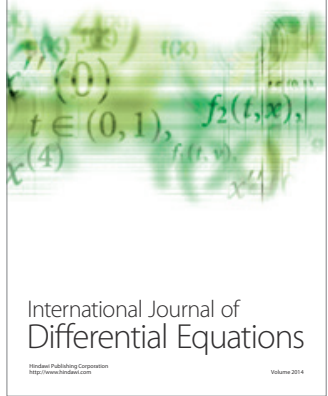
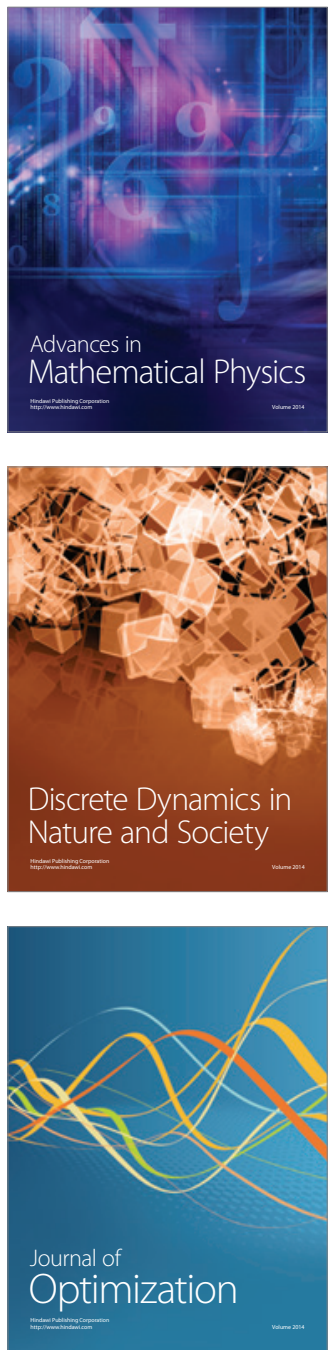